\title{
Unified quantum theory of elastic and inelastic atomic scattering from a physisorbed monolayer solid
}

\author{
Bruch, L. W.; Hansen, Flemming Yssing; Dammann, Bernd
}

Published in:

Physical Review B

Link to article, DOI:

10.1103/PhysRevB.95.214303

Publication date:

2017

Document Version

Publisher's PDF, also known as Version of record

Link back to DTU Orbit

Citation (APA):

Bruch, L. W., Hansen, F. Y., \& Dammann, B. (2017). Unified quantum theory of elastic and inelastic atomic scattering from a physisorbed monolayer solid. Physical Review B, 95(21), [214303].

https://doi.org/10.1103/PhysRevB.95.214303

\section{General rights}

Copyright and moral rights for the publications made accessible in the public portal are retained by the authors and/or other copyright owners and it is a condition of accessing publications that users recognise and abide by the legal requirements associated with these rights.

- Users may download and print one copy of any publication from the public portal for the purpose of private study or research.

- You may not further distribute the material or use it for any profit-making activity or commercial gain

- You may freely distribute the URL identifying the publication in the public portal 


\title{
Unified quantum theory of elastic and inelastic atomic scattering from a physisorbed monolayer solid
}

\author{
L. W. Bruch \\ Department of Physics, University of Wisconsin-Madison, 1150 University Avenue, Madison WI 53706, USA \\ F. Y. Hansen* \\ Department of Chemistry, Technical University of Denmark, IK-207-DTU, DK-2800 Lyngby, Denmark \\ B. Dammann \\ Department of Applied Mathematics and Computer Science, Technical University of Denmark, 324-DTU, DK 2800 Lyngby, Denmark
}

(Received 28 December 2016; published 12 June 2017)

\begin{abstract}
A unified quantum theory of the elastic and inelastic scattering of low energy He atoms by a physisorbed monolayer solid in the one-phonon approximation is given. It uses a time-dependent wave packet with phonon creation and annihilation components and has a self-consistent feedback between the wave functions for elastic and inelastic scattered atoms. An attenuation of diffraction scattering by inelastic processes thus is inherent in the theory. The atomic motion and monolayer vibrations in the harmonic approximation are treated quantum mechanically and unitarity is preserved. The evaluation of specific one-phonon events includes contributions from diffuse inelastic scattering in other phonon modes. Effects of thermally excited phonons are included using a mean field approximation. The theory is applied to an incommensurate $\mathrm{Xe} / \mathrm{Pt}(111)$ monolayer (incident energy $\left.E_{i}=4-16 \mathrm{meV}\right)$, a commensurate Xe/graphite monolayer $\left(E_{i} \simeq 64 \mathrm{meV}\right)$, and an incommensurate $\mathrm{Xe} / \mathrm{Cu}(001)$ monolayer $\left(E_{i} \simeq 8 \mathrm{meV}\right)$. The monolayers are very corrugated targets and there are transient closed diffraction and inelastic channels in the calculations. In many cases, the energy gain events have strengths comparable to the energy loss events.
\end{abstract}

DOI: 10.1103/PhysRevB.95.214303

\section{INTRODUCTION}

As the capability of helium atom scattering (HAS) experiments to measure the phonon spectra of physisorbed monolayers has progressed [1,2], there has been a corresponding need to refine the theory of the inelastic excitation process [3-5]. Because the monolayers are quite corrugated targets, the experiments frequently include combined effects of strong diffraction and a continuum of inelastic phonon creation and annihilation events. Experiments for inert gas monolayers [4,6-9] detected dispersive "in-plane" phonon branches but with the strongest signals for a relatively low beam energy $E_{i} \simeq 8 \mathrm{meV}$. Under these conditions, the probe atom wave function is deformed by many phonon processes simultaneously. We develop a unified quantum theory for the elastic and inelastic scattering that incorporates this picture.

Helium atom beams with still lower energies, below $3 \mathrm{meV}$, have been demonstrated in recent work [10,11]. This is an extreme quantum regime for atomic scattering and relative diffraction intensities accompanying quantum reflection have been reported [10]. However, much quantitative analysis of diffraction intensities for beams of energy $E_{i}<10$ meV relies on elementary Debye-Waller attenuation approximations [12]. Similar approximations are used in analyzing specular helium reflection for information on graphene-metal bonding [13]. There has been progress in the calculation of diffraction intensities with explicit coupling to inelastic channels [14] and with multichannel elastic scattering theory for heavier atoms [15]. However, a difficult combination of diffraction

*Corresponding author: flemming@kemi.dtu.dk and strong inelastic scattering occurs in experiments for physisorbed monolayers.

We present the one-phonon approximation to a unified self-consistent theory $\mathrm{SC}(T)$ of elastic and inelastic scattering of helium at low $E_{i}$. The target is a harmonic monolayer solid at temperatures $T$ where many modes have appreciable thermal-average phonon occupation numbers but where single phonon creation and annihilation events dominate the inelastic scattering. The calculations include properties such as the total elastic strength, the specular elastic strength, and the one-phonon creation and annihilation strengths. Results are compared with those from a more approximate NSC theory [5] in which thermal effects are estimated by multiplying the scattering strengths for an initially static target by factors based on the phonon population and a harmonic approximation to the Debye-Waller factor.

The theory is illustrated with three examples from HAS experiments with an adsorbed monolayer xenon solid, $\mathrm{Xe} / \mathrm{Pt}(111)$ Ref. [7], Xe/Cu(001) Ref. [8], and Xe on the basal plane of graphite Ref. [16]. For these examples, good independently determined interaction models are available, but the targets are relatively soft and the inelastic effects are large.

The paper is organized as follows: Section II has a brief review of theoretical work, with an emphasis on inert gas targets; Sec. III presents the self-consistent theory of the elastic and inelastic scattering, including an approximation for a thermal target. Section IV contains a summary of models and computational methods used here. The three examples are presented in Sec. V. Section VI contains our conclusions. The Appendix has a summary of the NSC approximation. Further computational results and amplifications of the discussion in the text are presented in the Supplemental Material [17]. 


\section{HELIUM SCATTERING BY INERT GAS MONOLAYERS}

The use of atomic scattering as a probe of surface structure and dynamics has a long history [18] but, because of the strong coupling in the scattering event, implementation of quantitative theoretical analysis of the intensities in elastic and inelastic channels remains a formidable problem $[3,19]$. The strong coupling is manifested in the relatively small fraction of the scattering that is in elastic channels and in symmetry-breaking effects such as the excitation of shear horizontal $(\mathrm{SH})$ phonons for scattering near high-symmetry directions of a monolayer solid [5]. The applicability of the traditional Debye-Waller formulation of attenuation of elastic (diffraction) intensities by inelastic processes is rather limited [20] for a low energy atom probe, and for heavy inert gas atoms there has been major recent development of the theory $[21,22]$. The intensity of one-phonon creation events may even increase with temperature, contrary to the simple language of Debye-Waller attenuation [23].

For atomic diffraction by xenon monolayer targets, the theoretical work using independently determined pair potential models began with close-coupling calculations [24-27] that did not include the inelastic terms self-consistently. This was followed by general formulations [28] of the diffraction calculation that incorporate the effect of inelastic scattering by a complex optical (absorbing) potential for the propagation of the elastic wave function. There also are more tractable versions [29] based on an inelastic close-coupling formulation and applied to cases with weak target corrugation or nearclassical approximations to the target motions. Another unitary formulation [30], that has been implemented recently [14] for helium scattering by a semimetal, estimates the effect of the inelastic bath by a close-coupling calculation that couples the diffraction channels to a representative inelastic channel [31] and then averages over a spectrum of choices for the representative channel. Diffuse inelastic scattering processes were included [32] in a close-coupling calculation for $\mathrm{He} / \mathrm{Xe} / \mathrm{Cu}(111)$ but without diffraction terms.

The theory applicable to phonon spectroscopies started with treatments of weakly corrugated surfaces, such as excitation of Rayleigh phonons on metal surfaces [33]. That analysis was in the context of the distorted wave Born approximation (DWBA). The inelastic scattering [34] of He from inert gas layers on $\operatorname{Ag}(111)$ and the availability of good He-inert gas pair potential models stimulated calculations of inelastic scattering with unitary approximations [35,36]. Later work [4] for $\mathrm{He} / \mathrm{Xe} / \mathrm{Cu}(001)$ simulated time-of-flight spectra but did not include diffraction effects. We used [5] an analogy to the perturbation theory formulation of inelastic neutron scattering [23] to treat the inelastic scattering from inert gas monolayers on $\operatorname{Pt}(111)$.

Several special features of low energy helium atom scattering (HAS) theory were included in previous calculations [37,38]. The force on the helium arises from several monolayer atoms (Armand effect), and this was incorporated by using phonon coordinates for the monolayer solid. The attractive atom-monolayer and atom-substrate potentials accelerate the atom (Beeby effect), and this was included in the dynamics of wave packet propagation. The attenuation of elastic strengths by phonon creation was estimated with a harmonic approximation for the Debye-Waller factor. A summary is given in the Appendix.

Nevertheless, it is desirable to have a unified theory of the elastic and inelastic scattering of low energy atoms that meets the following requirements: (i) The projectile (e.g., He) motion and the surface vibrations are treated fully quantum mechanically. (ii) Unitarity is preserved. The sum of all scattering probabilities, elastic and inelastic, must equal unity. (iii) The calculation of the inelastic scattering incorporates the diffuse scattering [39] arising from all modes in the Brillouin zone. (iv) The increasing importance of multiphonon processes at higher helium beam energies is included [40]. Gumhalter [3] presented a time-independent scattering theory that addresses these requirements and is an inelastic close-coupling theory.

In this paper we present a unified time-dependent theory based on wave-packet propagation that meets requirements (i) (iii). The projectile motion and surface vibrations are described quantum mechanically and unitarity is preserved through the scattering event. The theory allows for strong corrugation in the projectile-target potential energy surface and applies to harmonic monolayer solid targets for projectile energies in the one-phonon scattering regime.

Excitation and de-excitation of other phonon modes, the diffuse inelastic scattering, accompanies a particular specified phonon mode. The coupling of the wave functions for elastic and inelastic scattering is evaluated self-consistently for the given interaction potentials. Thus, attenuation effects are included for both elastic and inelastic channels. The channels are labeled by reciprocal lattice vectors $\mathbf{G}$ of the monolayer, and many $\mathbf{G}$ terms contribute to the scattering. There is a close formal correspondence to the inelastic close-coupling theory. The present time-dependent theory enables monitoring the evolution of the scattering event. In future, it may be extended to treating the thermal equilibration of the target system $[35,36]$.

\section{THEORY}

\section{A. Pure state harmonic solid target}

We develop the theory for a harmonic monolayer solid target that initially is in a pure state $\Phi_{i}$ characterized by phonon occupation numbers $n_{\mathbf{q}}$, where the composite index $\mathbf{q}=(\mathbf{Q}, \lambda)$ denotes the mode wave vector $\mathbf{Q}$ and polarization $\lambda$. Both energy loss and energy gain inelastic scattering of the incident atom (which we take to be ${ }^{4} \mathrm{He}$, for explicitness) may occur. The initial wave function $\Psi(t)$ of the projectile-monolayer system is a product of the He-atom state $\psi_{0}(t)$ and the monolayer state $\Phi_{i}$ :

$$
\Psi(t)=\psi_{0}(t) \Phi_{i}
$$

The initial $\psi_{0}$ consists of a Gaussian wave packet [5] for the motion in the $z$ direction ( $\hat{z}$, perpendicular to the surface) and a plane wave for the lateral motion

$$
\begin{aligned}
\psi_{0}(\mathbf{R}, z, t=0)= & \left(\frac{2 A}{\pi}\right)^{1 / 4} \exp \left(i\left[k_{i z}\left(z-z_{0}\right)+\mathbf{K}_{\|} \cdot \mathbf{R}\right]\right) \\
& \times \exp \left(-A\left[z-z_{0}\right]^{2}\right)
\end{aligned}
$$

The incident energy of the packet $E_{i}=\hbar^{2}\left(k_{i z}^{2}+\mathbf{K}_{\|}^{2}+A\right) /$ $2 m_{\mathrm{He}}$ includes the small spread $(\propto \sqrt{A})$ about $k_{i z}$, the central wave vector component along $\hat{z}$. $\mathbf{K}_{\|}$is the lateral wave vector, 
$\mathbf{k}_{i}=\mathbf{K}_{\|}+k_{i z} \hat{z}$, and $m_{\mathrm{He}}$ the mass of the atom. The constant $A$ determines how monochromatic the incident wave is; the initial packet center $z_{0}$ is placed so that the atom is well outside the range of forces from the monolayer and substrate. During the scattering, the packet deforms and coupled channels $\mathbf{K}_{\|}+\mathbf{G}$ are populated.

The initial monolayer state is a product of orthonormal states $\left|n_{\mathbf{q}}\right\rangle, \Phi_{i}=\Pi_{\mathbf{q}}\left|n_{\mathbf{q}}\right\rangle$, where the integer $n_{\mathbf{q}}$ is the number of phonons in that mode. The wave vectors $\mathbf{Q}$ lie in the first Brillouin zone of the monolayer lattice or an equivalent unit cell.

The evolution of the scattering event is governed by the time-dependent Schrödinger equation for the total wave function

$$
i \hbar \frac{\partial \Psi(t)}{\partial t}=\hat{H} \Psi(t)
$$

with the Hamiltonian $\hat{H}=\hat{K}_{\text {kin }}+\hat{H}_{\text {ph }}+V_{c}$, where

$\hat{K}_{\text {kin }}=-\frac{\hbar^{2}}{2 m_{\mathrm{He}}} \nabla_{\mathrm{He}}^{2} ; \quad$ kinetic energy operator for $\mathrm{He}$

$\hat{H}_{\mathrm{ph}}=\sum_{\mathbf{q}} \hbar \omega(\mathbf{q})\left[\hat{a}^{\dagger}(\mathbf{q}) \hat{a}(\mathbf{q})-n_{\mathbf{q}}\right] ;$ phonon energy operator

$$
V_{c}=\sum_{j} u\left(\left|\mathbf{r}_{\mathrm{He}}-\mathbf{r}_{j}\right|\right) ; \quad \text { He-monolayer interaction. }
$$

Here $\hbar \omega(\mathbf{q})$ is the phonon energy and $\hat{H}_{\mathrm{ph}}$ is the shift in monolayer energy relative to its initial value as a result of the interaction with He. $\hat{a}^{\dagger}(\mathbf{q})$ and $\hat{a}(\mathbf{q})$ are the phonon creation and annihilation operators, respectively. $n_{\mathbf{q}}$ is the (initial) eigenvalue of the number operator $\hat{n}_{\mathbf{q}} \equiv \hat{a}^{\dagger}(\mathbf{q}) \hat{a}(\mathbf{q}) . \mathbf{r}_{\mathrm{He}}$ is the position of the He atom; $\mathbf{r}_{j}$ is the position of the monolayer atom $j . \mathbf{r}_{j}$ is the sum of the static equilibrium position $\mathbf{r}_{j}^{\mathrm{eq}}$ and the dynamic displacement $\rho_{j}$ (e.g., due to vibrational motion), $\mathbf{r}_{j}=\mathbf{r}_{j}^{\mathrm{eq}}+\boldsymbol{\rho}_{j}$. The pair-sum interaction potential $V_{c}$ is expanded to first order in $\boldsymbol{\rho}_{j}$ and is written as a sum of a static part $V_{s}\left(\mathbf{r}_{\mathrm{He}}\right)$ and a dynamic part $V_{1}\left(\mathbf{r}_{\mathrm{He}}\right)$

$$
\begin{aligned}
V_{c} & \simeq \sum_{j} u\left(\left|\mathbf{r}_{\mathrm{He}}-\mathbf{r}_{j}^{\mathrm{eq}}\right|\right)+\sum_{j} \nabla_{j} u\left(\left|\mathbf{r}_{\mathrm{He}}-\mathbf{r}_{j}\right|\right)_{\left(\mathbf{r}_{j}=\mathbf{r}_{j}^{\mathrm{eq}}\right)} \cdot \boldsymbol{\rho}_{j} \\
& \equiv V_{s}\left(\mathbf{r}_{\mathrm{He}}\right)+V_{1}\left(\mathbf{r}_{\mathrm{He}}\right) .
\end{aligned}
$$

(We add the long-range attraction $-C_{3} / z^{3}$ between the He and substrate to $V_{s}$.) The strong coupling character of the HAS event is retained, but the wave function basis adopted in Eq. (8) limits the inelastic transfer to one-phonon processes [41].

The expansion of $\boldsymbol{\rho}_{j}$ in phonon operators is [42], with $\mathbf{R}_{j}^{\mathrm{eq}}$ the $2 \mathrm{D}$ projection of $\mathbf{r}_{j}^{\mathrm{eq}}$,

$$
\boldsymbol{\rho}_{j}=\sum_{\mathbf{q}} \sqrt{\frac{\hbar}{2 m N \omega(\mathbf{q})}}\left[\hat{a}(\mathbf{q})+\hat{a}^{\dagger}(-\mathbf{q})\right] \exp \left(i \mathbf{Q} \cdot \mathbf{R}_{j}^{\mathrm{eq}}\right) \hat{\mathbf{e}}(\mathbf{q}),
$$

where $N$ is the number of atoms of mass $m$ in the monolayer and $\hat{\mathbf{e}}(\mathbf{q})$ is the polarization unit vector. For a monatomic Bravais lattice there are $3 N \mathbf{q}^{\prime} \mathrm{s}$ in the sum. The dynamic part of the interaction potential has the form

$$
V_{1}\left(\mathbf{r}_{\mathrm{He}}\right)=\sum_{\mathbf{q}} V(\mathbf{q}) \hat{a}(\mathbf{q})+V^{*}(\mathbf{q}) \hat{a}^{\dagger}(\mathbf{q}),
$$

where the coefficients $V(\mathbf{q})$ and their complex conjugates $V^{*}(\mathbf{q})$ depend [5] on the potentials $u\left(\left|\mathbf{r}_{\mathrm{He}}-\mathbf{r}_{j}\right|\right)$ and the phonon variables of Eq. (6).

The time-dependent wave function $\Psi(t)$ becomes a superposition of the elastic scattering wave function $\psi_{0}(t) \Phi_{i}$ and $6 N$ inelastic scattering wave functions specifying phonon creation and annihilation, $\psi_{1}^{+}(\mathbf{q}, t) \hat{a}^{\dagger}(\mathbf{q}) \Phi_{i}$ and $\psi_{1}^{-}(\mathbf{q}, t) \hat{a}(\mathbf{q}) \Phi_{i}$,

$$
\begin{aligned}
\Psi(t)= & \psi_{0}(t) \Phi_{i}+\sum_{\mathbf{q}} \psi_{1}^{+}(\mathbf{q}, t) \hat{a}^{\dagger}(\mathbf{q}) \Phi_{i} \\
& +\sum_{\mathbf{q}} \psi_{1}^{-}(\mathbf{q}, t) \hat{a}(\mathbf{q}) \Phi_{\mathbf{i}} .
\end{aligned}
$$

$\psi_{1}^{+}(\mathbf{q}, t)$ and $\psi_{1}^{-}(\mathbf{q}, t)$ denote the He-atom wave functions associated with mode q. Using $\Psi(t)$ in Eq. (3) and projecting the result onto $\Phi_{i}, \hat{a}^{\dagger}(\mathbf{q}) \Phi_{i}$, and $\hat{a}(\mathbf{q}) \Phi_{i}$, respectively, gives a coupled system of equations of motion:

$$
\begin{aligned}
i \hbar \frac{\partial \psi_{0}(t)}{\partial t}= & {\left[\hat{K}_{\mathrm{He}}+V_{s}\right] \psi_{0}(t)+\sum_{\mathbf{q}}\left(1+n_{\mathbf{q}}\right) V^{*}(\mathbf{q}) \psi_{1}^{+}(\mathbf{q}, t) } \\
& +\sum_{\mathbf{q}} n_{\mathbf{q}} V(\mathbf{q}) \psi_{1}^{-}(\mathbf{q}, t), \\
i \hbar \frac{\partial \psi_{1}^{+}(\mathbf{q}, t)}{\partial t}= & {\left[\hat{K}_{\mathrm{He}}+V_{s}+\hbar \omega(\mathbf{q})\right] \psi_{1}^{+}(\mathbf{q}, t)+V(\mathbf{q}) \psi_{0}(t), } \\
i \hbar \frac{\partial \psi_{1}^{-}(\mathbf{q}, t)}{\partial t}= & {\left[\hat{K}_{\mathrm{He}}+V_{s}-\hbar \omega(\mathbf{q})\right] \psi_{1}^{-}(\mathbf{q}, t)+V^{*}(\mathbf{q}) \psi_{0}(t) . }
\end{aligned}
$$

The norm of the wave function is given by

$$
\begin{aligned}
\left\langle\Psi^{*}(t) \Psi(t)\right\rangle= & \left\langle\left|\psi_{0}(t)\right|^{2}+\sum_{\mathbf{q}}\left(1+n_{\mathbf{q}}\right)\left|\psi_{1}^{+}(\mathbf{q}, t)\right|^{2}\right. \\
& \left.+\sum_{\mathbf{q}} n_{\mathbf{q}}\left|\psi_{1}^{-}(\mathbf{q}, t)\right|^{2}\right\rangle .
\end{aligned}
$$

Equations (9) imply that the time derivative of the norm in Eq. (10) is equal to zero at all times; i.e., the norm is conserved, $\left\langle\Psi^{*}(t) \Psi(t)\right\rangle=1$, and the theory is unitary.

Equations (9) have a ladder character arising from the one-phonon inelastic processes and a configuration with occupation number $n_{\mathbf{q}}$ is coupled to $n_{\mathbf{q}} \pm 1$. The ladder may be extended to terms $n_{\mathbf{q}} \pm 2$ by adding such configurations to the wave function Eq. (8). Thus this formulation has much overlap with one used by Kraus et al., [14] although we treat several q simultaneously while they assume only one mode is active in the scattering process.

In the scattering from a monolayer (2D) solid, the channels are specified by reciprocal lattice vectors $\mathbf{G}$ of the target. We expand $\psi_{0}(t), \psi_{1}^{+}(\mathbf{q}, t)$ and $\psi_{1}^{-}(\mathbf{q}, t)$ as sums over $N_{G}$ Gchannels and construct the equations of motion for these components. With $\mathbf{r}_{\mathrm{He}}$ expressed as a lateral vector $\mathbf{R}$ and distance $z$ along $\hat{z}, \mathbf{r}_{\mathrm{He}}=\mathbf{R}+z \hat{z}$, the potential energy terms are

$$
\begin{aligned}
V_{s}(\mathbf{R}, z) & =V(0, z)+\sum_{\mathbf{G} \neq 0} V(\mathbf{G}, z) \exp (i \mathbf{G} \cdot \mathbf{R}) \\
V(\mathbf{q}) & =\sum_{\mathbf{G}} C_{\lambda}(\mathbf{G}+\mathbf{Q}, z) \exp (i[\mathbf{G}+\mathbf{Q}] \cdot \mathbf{R}),
\end{aligned}
$$


and the functions $\psi_{0}(t), \psi_{1}^{+}(\mathbf{q}, t)$, and $\psi_{1}^{-}(\mathbf{q}, t)$ are, with initial lateral wave vector $\mathbf{K}_{\|}$:

$$
\begin{aligned}
\psi_{0}(t) & =\sum_{\mathbf{G}} \psi(\mathbf{G}, z, t) \exp \left(i\left[\mathbf{K}_{\|}+\mathbf{G}\right] \cdot \mathbf{R}\right) \\
\psi_{1}^{+}(\mathbf{q}, t) & =\sum_{\mathbf{G}} S_{\mathbf{q}}^{+}(\mathbf{G}, z, t) \exp \left(i\left[\mathbf{K}_{\|}-\mathbf{Q}+\mathbf{G}\right] \cdot \mathbf{R}\right) \\
\psi_{1}^{-}(\mathbf{q}, t) & =\sum_{\mathbf{G}} S_{\mathbf{q}}^{-}(\mathbf{G}, z, t) \exp \left(i\left[\mathbf{K}_{\|}+\mathbf{Q}+\mathbf{G}\right] \cdot \mathbf{R}\right) .
\end{aligned}
$$

The final coupled equations of motion are

$$
\begin{aligned}
i \hbar \frac{\partial \psi(\mathbf{G}, z, t)}{\partial t}= & \frac{\hbar^{2}}{2 m_{\mathrm{He}}}\left[\left(\mathbf{K}_{\|}+\mathbf{G}\right)^{2}-\frac{\partial^{2}}{\partial z^{2}}\right] \psi(\mathbf{G}, z, t)+\sum_{\mathbf{G}^{\prime}} V\left(\mathbf{G}-\mathbf{G}^{\prime}, z\right) \psi\left(\mathbf{G}^{\prime}, z, t\right) \\
& +\sum_{\mathbf{q}}\left(1+n_{\mathbf{q}}\right) \sum_{\mathbf{G}^{\prime}} C_{\lambda}^{*}\left(\mathbf{G}-\mathbf{G}^{\prime}+\mathbf{Q}, z\right) S_{\mathbf{q}}^{+}\left(\mathbf{G}^{\prime}, z, t\right)+\sum_{\mathbf{q}} n_{\mathbf{q}} \sum_{\mathbf{G}^{\prime}} C_{\lambda}\left(\mathbf{G}-\mathbf{G}^{\prime}-\mathbf{Q}, z\right) S_{\mathbf{q}}^{-}\left(\mathbf{G}^{\prime}, z, t\right) \\
i \hbar \frac{\partial S_{\mathbf{q}}^{+}(\mathbf{G}, z, t)}{\partial t}= & {\left[\frac{\hbar^{2}}{2 m_{\mathrm{He}}}\left(\left(\mathbf{K}_{\|}-\mathbf{Q}+\mathbf{G}\right)^{2}-\frac{\partial^{2}}{\partial z^{2}}\right)+\hbar \omega(\mathbf{q})\right] S_{\mathbf{q}}^{+}(\mathbf{G}, z, t)+\sum_{\mathbf{G}^{\prime}} V\left(\mathbf{G}-\mathbf{G}^{\prime}, z\right) S_{\mathbf{q}}^{+}\left(\mathbf{G}^{\prime}, z, t\right) } \\
& +\sum_{\mathbf{G}^{\prime}} C_{\lambda}\left(\mathbf{G}^{\prime}-\mathbf{G}+\mathbf{Q}, z\right) \psi\left(\mathbf{G}^{\prime}, z, t\right) \\
i \hbar \frac{\partial S_{\mathbf{q}}^{-}(\mathbf{G}, z, t)}{\partial t}= & {\left[\frac{\hbar^{2}}{2 m_{\mathrm{He}}}\left(\left(\mathbf{K}_{\|}+\mathbf{Q}+\mathbf{G}\right)^{2}-\frac{\partial^{2}}{\partial z^{2}}\right)-\hbar \omega(\mathbf{q})\right] S_{\mathbf{q}}^{-}(\mathbf{G}, z, t)+\sum_{\mathbf{G}^{\prime}} V^{*}\left(\mathbf{G}-\mathbf{G}^{\prime}, z\right) S_{\mathbf{q}}^{-}\left(\mathbf{G}^{\prime}, z, t\right) } \\
& +\sum_{\mathbf{G}^{\prime}} C_{\lambda}^{*}\left(\mathbf{G}^{\prime}-\mathbf{G}-\mathbf{Q}, z\right) \psi\left(\mathbf{G}^{\prime}, z, t\right) .
\end{aligned}
$$

Explicit expressions for the coefficients $V(\mathbf{G}, z)$ and $C_{\lambda}(\mathbf{G} \pm$ $\mathbf{Q}, z)$ in terms of the interaction potential are given in Ref. [5]. The equations to be solved extend our previous working equations [5,38] as follows: The elastic channel function in Eq. (13) is coupled to phonon creation and annihilation channel functions for all $\mathbf{q}$. For each $\mathbf{q}=(\mathbf{Q}, \lambda)$ in the sums in Eq. (13) there is a differential equation for $S_{\mathbf{q}}^{+}$, Eq. (14), for events that create a phonon of that $\mathbf{q}$ and one for $S_{\mathbf{q}}^{-}$, Eq. (15), for events that annihilate a phonon of that $\mathbf{q}$. The $\mathbf{q}$ sums in Eq. (13) include all polarizations $\lambda$ and all wave vectors $\mathbf{Q}$ in the first Brillouin zone; formally there is a continuum of $\mathbf{Q}$ which we approximate with a discrete set of $\mathbf{Q}^{\prime}$ s.

The first two terms on the right hand sides of Eqs. (13)-(15) give the coupled channel propagation of $\psi(\mathbf{G}, z, t), S_{\mathbf{q}}^{+}(\mathbf{G}, z, t)$, and $S_{\mathbf{q}}^{-}(\mathbf{G}, z, t)$, respectively. The final terms in $C_{\lambda}$ and $C_{\lambda}^{*}$ transfer norm between elastic and inelastic channels. Initially they serve as sinks and sources for the elastic and inelastic strengths, respectively, but they also include feedback effects that may restore elastic strength as the collision evolves. The factors $\left(n_{\mathbf{q}}+1\right)$ and $n_{\mathbf{q}}$ in the last two terms in Eq. (13) reflect the quantum mechanical asymmetry in the phonon creation and annihilation probabilities. There is no direct coupling between $S_{\mathbf{q}}^{ \pm}(\mathbf{G}, z, t)$ for different $\mathbf{q}$ modes, only an indirect coupling via $\psi(\mathbf{G}, z, t)$.

The coupling $V(\mathbf{q})$ in these equations is of the order of $1 / \sqrt{N}$. There are $6 N$ functions $\psi_{1}^{ \pm}(\mathbf{q})$ in Eq. (9) and in Eq. (13) the $S_{\mathrm{q}}^{ \pm}$lead to $O(1)$ changes of the elastic channel functions $\psi(\mathbf{G})$. In principle, this gives a full account of the 1-phonon diffuse background and can be implemented by a fine sampling of the Brillouin zone with $N_{Q}$ values of $\mathbf{Q}$. As with the truncation to $N_{G} \mathbf{G}$ channels, the sampling can be systematically improved.

However, the present $\mathbf{Q}$ grids are unlikely to include the phonon spectroscopy conditions leading to a particular phonon, to within experimental resolution. Therefore, to mimic a specific time-of-flight experiment, we add one more $\mathbf{Q}$, the wave vector of the particular inelastic scattering event, if it is not among the basic $\mathbf{Q}$ grid for the Brillouin zone. This additional mode $\mathbf{Q}$ then determines the incident angle $\theta_{i}$ for the scattering in an experiment with scan-curve constraints [1]. The basic $\mathbf{q}$ set [with $\omega(\mathbf{q})$ ] is independent of the particular scattering event. The effect of the $\mathbf{q}$ modes does depend on the scattering geometry that is determined by the $\mathbf{Q}$ and frequency $\omega(\mathbf{Q}, \lambda)$ of the chosen phonon.

\section{B. Thermal target}

The theoretical development of Sec. III A treats the scattering for a monolayer initially in an energy eigenstate characterized by a set of integer phonon occupation numbers $\left\{n_{\mathbf{q}}\right\}$. For a zero temperature target all the $n_{\mathbf{q}}$ are zero, but at finite temperatures the configurations have nonzero $n_{\mathbf{q}}$ 's. In principle, the elastic and inelastic strengths for a target at finite temperature are calculated as a canonical average of the scattering strengths for any combination of the number of phonons in each of the modes $\mathbf{q}$ [43]. In practice, such a formulation leads directly to a well-known exponential scaling of the calculation [44].

A target at temperature $T$ has average occupation numbers $\bar{n}_{\mathbf{q}}$ given by

$$
\bar{n}_{\mathbf{q}}=\frac{1}{\exp \left(\hbar \omega_{\mathbf{q}} / k_{\mathrm{B}} T\right)-1} .
$$

To make the treatment of a thermal target tractable, we use the values $\left\{\bar{n}_{\mathbf{q}}\right\}$ in place of $\left\{n_{\mathbf{q}}\right\}$ in Eqs. (13) to (15). This provides for both phonon annihilation and creation events [45].

Replacing the occupation numbers by thermal average occupation numbers and the multiple configurations of a 
thermal ensemble by a single configuration has the appearance of a mean-field approximation. Related approximations using the thermal average are included in the time-independent scattering theory of Gumhalter and co-workers $[3,4]$, the characterization of the inelastic bath in a sticking calculation by Medina and Jackson [46], and implicitly in the calculation of the attenuation of diffraction by Kraus et al. [14]. We believe this includes major aspects of the thermally excited target for low energy HAS. It incorporates a very different picture than that used for the thermal effects in a dynamic low energy electron diffraction (LEED) calculation, where the incident electron is assumed to scatter from a random instantaneous configuration of the target $[47,48]$. The speed of a $20 \mathrm{meV}$ $\mathrm{He}$ atom is slower by a factor of $6 \times 10^{3}$ than that of a $100 \mathrm{eV}$ electron and the period of a lattice vibration of monolayer xenon is on the same scale as the interaction time of the atom and monolayer.

Our emphasis is on the phonon creation (energy loss) events because they were the best resolved in the HAS experiments on monolayer xenon [1]. In recent applications of HAS to more complex monolayers at higher temperature [49] the energy gain events are more evident. Indeed, there are many cases in the present work where the strengths of energy gain and energy loss events are comparable.

\section{Formulation of results}

The targets are 2D Bravais lattices with reciprocal lattice vectors $\mathbf{G}$. The branches of the monolayer phonon spectrum are labeled SH (shear horizontal), LA (longitudinal acoustic), and $S$ ( $\hat{z}$ or perpendicular vibration), using the dominant polarization of the branch.

The scattering calculations yield the norms (strengths) of the wave functions in the various elastic and inelastic channels; because the total wave function is normalized, the sum of these strengths is 1 . We adopt the following notation: $N_{\mathrm{E}}$ is the total probability of elastic scattering, that is, the sum of the specular $(\mathbf{G}=0)$ elastic strength $N_{0}$ and the strength in the diffraction $(\mathbf{G} \neq 0)$ peaks. The total diffuse inelastic scattering is separated into the total diffuse strengths for phonon creation $\mathrm{DI}^{+}$and annihilation $\mathrm{DI}^{-}$. When the excitation of a specific mode is treated by adding another $\mathbf{Q}$ to the diffuse set, the total additional one-phonon inelastic strengths for creation and annihilation are $Q I^{+}$and $Q I^{-}$, respectively, and the additional specular inelastic strengths are $Q I_{0}^{+}$and $Q I_{0}^{-}$. The $Q I_{0}^{+}$and $Q I_{0}^{-}$depend on the number $N_{Q}$ of wave vectors used to sample the Brillouin zone. The ratios $Q I_{0}^{+} / Q I^{+}$and $Q I_{0}^{-} / Q I^{-}$, in which the dependence on $N_{Q}$ mostly cancels, are measures of how much of that process is concentrated in the specular channel. All these functions depend on the temperature $T$.

These definitions differ from components of the experimental differential reflection coefficient [50] only by a phase space factor $k_{f} / k_{i z}$, where $k_{f}$ is the final wave number of the scattered He and $k_{i z}$, Eq. (2), sets the incident flux. Note that the superscripts + and - refer to inelastic processes in which monolayer phonons are created and annihilated, respectively; the corresponding energy changes for the He are energy loss and gain, i.e., they are opposite to the $+/-$ of the calculations.

\section{IMPLEMENTATION: MODELS AND METHODS}

\section{A. Models}

We use the well-documented HFD-B2 potentials [27,51] for the $\mathrm{He}-\mathrm{Xe}$ and $\mathrm{Xe}-\mathrm{Xe}$ isolated pair potentials. The normal modes (frequency and polarization) of the monolayer solid at wave vector $\mathbf{Q}$ are determined in lattice dynamics calculations. The interaction of the He atom with the supporting $\operatorname{Pt}(111)$, $\mathrm{Cu}(001)$, or graphite substrate is given by the nonretarded van der Waals energy $-C_{3} / z^{3}$ with semiempirical values for $C_{3}$. We neglect effects of the dynamical coupling of the monolayer to the substrate; this may be a significant idealization because a hybridization of the monolayer S mode and the substrate Rayleigh wave is observed near the Brillouin zone center for the three examples $[4,7,52]$. We assume uniform monolayer triangular lattices with no mass-density-wave distortions.

The HFD-B2 model for $\mathrm{He}-\mathrm{Xe}$ is the basis for the calculation of selective adsorption energy levels for $\mathrm{He} / \mathrm{Xe} /$ graphite [27]; we omit the many-body interactions included in fitting such energy levels closely [26,27]. The corresponding results for $\mathrm{He} / \mathrm{Xe} / \mathrm{Pt}(111)$ are given in Ref. [38] while measures of the corrugation of the $\mathrm{He} / \mathrm{Xe} / \mathrm{Pt}(111)$ potential energy surface are given in Ref. [53]. Modeling of monolayer $\mathrm{Xe} /$ graphite using the HFD-B2 Xe-Xe potential is reported in Ref. [54] and of $\mathrm{Xe} / \mathrm{Pt}(111)$ in Ref. [7].

We characterize [38] the corrugation of the potential energy surface $V_{s}(\mathbf{R}, z)$ by the differences in the heights at which $V_{S}(\mathbf{R}, z)$ is equal to $E$ for three lateral positions: $z_{C}$ at the threefold hollow site, $z_{B}$ at the bridge site, and $z_{A}$ at the atop site, $\left[z_{B C}=z_{B}-z_{C}\right.$ and $\left.z_{A C}=z_{A}-z_{C}\right]$ and by the reciprocal lattice shell number JG which suffices to make $\left|V\left(G, z_{C}\right) / V\left(G_{0}, z_{C}\right)\right|<0.001$. The latter condition is a measure of the convergence of the series in Eq. (11).

In the $\mathrm{He} / \mathrm{Xe} / \mathrm{Pt}(111)$ experiments [7], the target is a compressed triangular lattice with nearest-neighbor spacing $L_{n n}=4.33 \AA$. We supplement the Xe-Xe potential with the McLachlan substrate-mediated dispersion energy with $\operatorname{Pt}(111)$ coefficients $C_{s 1}=201$ a.u., $C_{s 2}=154$ a.u., and overlayer distance $L_{o v}=2.2 \AA$ as defined in Refs. [7,42]. The He$\operatorname{Pt}(111)$ van der Waals energy coefficient is $C_{3}=0.0632$ a.u. The $\mathrm{S}$-mode energy is $\hbar \omega_{\perp}=3.5 \mathrm{meV}$. For this model the corrugation measures for contours of energy $10 \mathrm{meV}$ and $20 \mathrm{meV}$ are $z_{B C} \simeq 0.23 \AA, z_{A C} \simeq 0.94 \AA$, and $\mathrm{JG}=5$.

For $\mathrm{He} / \mathrm{Xe} / \mathrm{Cu}(001)$, Sec. V B, the substrate terms are $C_{3}=$ 0.0583 a.u., $C_{s 1}=191$ a.u., $C_{s 2}=146$ a.u., and $L_{o v}=2.45 \AA$ from Ref. [7]. The S-mode energy is [4] $\hbar \omega_{\perp}=2.70 \mathrm{meV}$. For the $E=10 \mathrm{meV}$ contour, $z_{B C}$ increases from 0.24 to $0.27 \AA$ and $z_{A C}$ from 0.99 to $1.07 \AA$ as $L_{n n}$ increases from 4.41 to $4.52 \AA$ (the range in the experiments); $\mathrm{JG}=6$.

For $\mathrm{He} / \mathrm{Xe} /$ graphite, $\mathrm{Sec} . \mathrm{V} \mathrm{C}$, the substrate terms are $C_{3}=$ 0.043 a.u., $C_{s 1}=142$ a.u., $C_{s 2}=89$ a.u., and $L_{o v}=1.9 \AA$. That xenon monolayer is commensurate [16] with $L_{n n}=$ $4.26 \AA$ and its zone-center gap energy is $\hbar \omega_{0 \|}=0.66 \mathrm{meV}$ estimated from a model [54] for the Xe-graphite corrugation. The $\mathrm{S}$-mode energy is $[7,55] \hbar \omega_{\perp}=3.0 \mathrm{meV}$. The corrugation measures for the $E=63.8 \mathrm{meV}$ contour are $z_{B C} \simeq 0.23 \AA$, $z_{A C} \simeq 0.95 \AA$, and $\mathrm{JG}=6$. The height differences are close to the values for the corrugated hard wall fit by Bracco et al. [16] to their diffraction data, $\Delta z_{B C} \simeq 0.23 \AA$ and $\Delta z_{A C} \simeq 0.88 \AA$. (For the HFD-1 model used by Hutson and Schwartz [24,58], 
the measures are $z_{B C} \simeq 0.24 \AA, z_{A C} \simeq 0.98 \AA$, and $\mathrm{JG}=8$, i.e., a somewhat more corrugated surface with slower convergence for the $V_{G}$ series.)

\section{B. Methods}

The self-consistent (SC) time-dependent Schrödinger equation is solved numerically on a discrete grid in $z$ and for the many $\mathbf{G}$ and $\mathbf{q}$ channels. The numerical methods are the same as in Refs. [5,38]. The number of channel functions now is much larger, but the methods remain robust and enable precise solutions in this more complex calculation. The coupled linear differential equations for propagating the channel functions are solved using a fast Fourier transform for the kinetic energy operator and a simple stepping procedure for the time integration.

The total time span is long enough that transient trapping near the monolayer has decayed, but then some components of the wave functions reach the far end of the $z$ grid. To eliminate artifacts arising from reflections and transmissions, we add an absorbing optical potential there and calculate the flux of probability current into the absorber for each channel. When integrated over time this determines the absorbed strength in each channel [38]. The sum of the norm on the spatial grid and the total absorbed strength is constant to at least $1 / 10^{4}$; i.e., unitarity is preserved. In principle, all the norm is absorbed in the final state of the scattering event in the time-dependent theory; we treat cases where the final configurations have up to $90 \%$ of the norm absorbed and unitarity is still preserved this well. The addition of an absorbing potential complicates the time stepping because the algorithms for stable propagation with an imaginary (absorbing) potential and a real potential are different. Propagation of the wave function with an imaginary potential is stable in an algorithm using a first-order finite difference for the time derivative and unstable in one using a second-order difference for the time derivative; the reverse is true for the propagation with a real potential. Therefore a hybrid scheme is used with different propagation algorithms for the real and imaginary parts of the potential. Further details are given in Ref. [38].

We verify numerical convergence of the calculations by variation of $N_{\mathbf{G}}$ and $N_{\mathbf{Q}}$. The number $N_{\mathbf{G}}$ of reciprocal lattice vectors $\mathbf{G}$ (i.e., diffraction channels) in the calculation depends on the incident atom energy $E_{i}$ and increases with $E_{i}$.

$\mathbf{G}$ vectors are added until the results have converged to about $1 \%$. $N_{\mathbf{G}}$ typically is 46 for $E_{i}=4 \mathrm{meV}, 57$ for $8.2 \mathrm{meV}$, and 94 for $16.6 \mathrm{meV}$.

The Brillouin zone sum of the diffuse scattering is made with a fine grid of $N_{\mathbf{Q}}$ uniformly distributed $\mathbf{Q}$ and $N_{\mathbf{Q}}$ is used in place of $N$ in the factor $1 / \sqrt{N}$ in Eq. (6). Since the strength of a phonon excitation is proportional to $1 / N_{\mathbf{Q}}$ and the number of modes is $3 N_{\mathbf{Q}}$ (3 polarizations at each $\mathbf{Q}$ ), the total diffuse inelastic strength may become nearly independent of $N_{\mathbf{Q}}$ if $N_{\mathbf{Q}}$ is large enough. This also is an approximation that may be improved systematically; $N_{\mathbf{Q}}=15-35$ is large enough to give convergent results for the final diffraction strengths in our calculations.

The uniform grid of $\mathbf{Q}$ is constructed as follows. A parallelogram is formed from two primitive reciprocal lattice vectors $\mathbf{G}_{1}$ and $\mathbf{G}_{2}$ (of equal length and at $60^{\circ}$ for a triangular lattice). Adjacent sides are broken into $n_{\mathbf{Q}}$ equidistant intervals and the grid is set by the intersections of lines through these points and parallel to the other vector. The $\mathbf{Q}=0$ point is excluded to avoid the anomaly of vanishing $\omega$ at the Brillouin zone center of an incommensurate monolayer. Then the grid (denoted an $N_{Q}$ sampling) has

$$
N_{\mathbf{Q}}=n_{\mathbf{Q}}^{2}-1
$$

$\mathbf{Q}$ values, each with three polarizations. With $N_{\mathbf{G}}$ diffraction channels, there are $\left[N_{\mathbf{Q}} * 3 * 2\right] * N_{\mathbf{G}}$ functions $S_{\mathbf{q}}^{+}$and $S_{\mathbf{q}}^{-}$ for the diffuse inelastic scattering, $2 * N_{\mathbf{G}}$ for the specified single phonon that determined the scattering geometry, and $N_{\mathbf{G}}$ functions $\psi(\mathbf{G})$ for the elastic scattering, a total of $3 *\left(2 N_{\mathbf{Q}}+1\right) * N_{\mathbf{G}}$ differential equations and functions. This readily leads to a total of several thousand coupled functions in the calculations. In the simpler case of a target in its ground state, there are $\left(3 N_{\mathbf{Q}}+2\right) N_{\mathbf{G}}$ coupled functions. Most of the calculations had $N_{\mathbf{Q}}=15\left(n_{\mathbf{Q}}=4\right)$; all included the three polarizations ( $\mathrm{SH}, \mathrm{LA}$, and $\mathrm{S}$ ). To include the hybridization of the substrate Rayleigh mode and the monolayer $\mathrm{S}$ mode requires developing the coupled lattice dynamics and sampling Q's near the Brillouin zone center (with $N_{\mathbf{Q}}>35$ ); we have not done this modeling yet.

We apply the scan-curve geometry $[1,38]$ of experiments where the angle $\theta_{\mathrm{SD}}$ between the incoming beam and the scattered beam is fixed $\left[\theta_{\mathrm{SD}}=95.8^{\circ}\right.$ for the $\mathrm{He} / \mathrm{Xe} / \mathrm{Pt}(111)$ and $\mathrm{He} / \mathrm{Xe} / \mathrm{Cu}(001)$ experiments]. Adjusting the angle of incidence $\theta_{i}$ then leads to a scattered beam that enters the detector for the desired inelastic event or diffraction peak.

The wave packets Eq. (2) are the so-called polychromatic $(N W I=100)$ wave packets [38] that have an initial energy spread $\sigma_{E}=0.17 \sqrt{E_{i}}\left(=0.85 \mathrm{meV}\right.$ for $\left.E_{i}=25 \mathrm{meV}\right)$. In a few cases we used broader $(N W I=36)$ and narrower $(N W I=600)$ energy spreads to look for effects of spatial coherence of the wave packets; changes were generally smaller than $10 \%$.

\section{EXAMPLES}

\section{A. $\mathrm{Xe} / \mathrm{Pt}(111)$}

All three phonon branches were accessed in HAS experiments for inert gases on $\mathrm{Pt}(111)$ near high-symmetry directions, [7] where the three polarizations are "pure" $\mathrm{SH}$, LA, and S. The excitation of the SH branch was in apparent violation of a polarization selection rule, [8,23] but it could [5] arise from a small misalignment of the scattering plane relative to symmetry axes of the monolayer solid. We use a $2.6^{\circ}$ misalignment observed for $\mathrm{Xe} / \mathrm{Pt}(111)$ that arises from the Novaco-McTague orientational epitaxy [59].

The $\mathrm{He} / \mathrm{Xe} / \mathrm{Pt}(111)$ experiments [7] were performed at $50 \mathrm{~K}$ and with incident energies $E_{i}=8.2$ and $16.6 \mathrm{meV}$. We calculate for these conditions and include $E_{i}=4 \mathrm{meV}$ to explore whether that leads to enhanced sensitivity to the $\mathrm{SH}$ and LA branches. We also treat temperatures $T=25$ and $75 \mathrm{~K}$, which were accessible in the experiments, although no data were reported. The phonon wave vectors $\mathbf{Q}=-0.125 \AA^{-1}$ and $\mathbf{Q}=-0.25 \AA^{-1}$ in scattering planes at $2.6^{\circ}$ from the highsymmetry monolayer azimuths $\Gamma \mathrm{M}$ and $\Gamma \mathrm{K}$ are emphasized because the experiments showed strong specular inelastic 


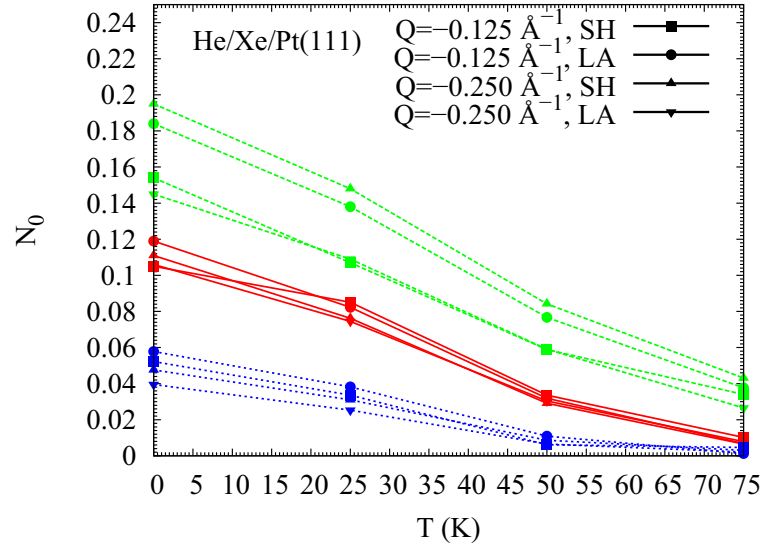

FIG. 1. Final specular elastic strengths (or fractions) $N_{0}$ as a function of temperature $T$ for two wave vectors $\mathbf{Q}$ and two polarizations SH and LA identified by the point symbols in the legend. The corresponding angles of incidence are given in Table I and Ref. [38]. The scattering plane is at $2.6^{\circ}$ to the monolayer $\Gamma \mathrm{M}$ azimuth. Energies $E_{i}$ are identified as dashed line (green) $4 \mathrm{meV}$, solid line (red) $8.2 \mathrm{meV}$, and dotted line (blue) $16.6 \mathrm{meV}$. The straight line segments are linear interpolations that join points corresponding to the same scattering geometry as guides to the eye. The Brillouin zone was sampled with $N_{\mathbf{Q}}=15$.

signals $Q I_{0}^{+}$at small $|\mathbf{Q}|$. The largest phonon energy for the xenon monolayer is about $5 \mathrm{meV}$; hence all phonon modes are thermally excited at $50 \mathrm{~K}$. Thus the effects on the scattering of varying incident energy $E_{i}$, temperature $T$, wave vector $\mathbf{Q}$, and phonon polarization are studied.

Figure 1 shows the elastic strength in the specular channel $N_{0}$ as a function of temperature $T$ and energy $E_{i}$ for four $\mathbf{q}$ cases with the scattering plane at $2.6^{\circ}$ to the $\Gamma M$ azimuth. The rather small values of $N_{0}$ at 8.2 and $16.6 \mathrm{meV}$ show how corrugated and soft the monolayer is. $N_{0}$ decreases steadily with increasing $T$; most of the reduction arises from inelastic scattering [17], but there also is a redistribution of intensity in diffraction channels.

Experiments frequently use the specular elastic strength as a measure of coverage. A normalized [relative to clean $\mathrm{Pt}(111)$ ] value of $0.04-0.05$ was observed [60] along $\Gamma \mathrm{K}$ for the monolayer at $E_{i}=5.3 \mathrm{meV}, \theta_{i}=47.9^{\circ}$, and $50 \mathrm{~K}$. Extrapolating (not shown here) the calculated values near $\Gamma \mathrm{K}$ for $E_{i}=4 \mathrm{meV}$ and $50 \mathrm{~K}$ to this angle gives $N_{0} \approx 0.04$.

Figure 2 shows the specular inelastic strength $Q I_{0}^{+}$for the same cases as in Fig. 1. For a given $\mathrm{SH}$ case, $Q \mathrm{I}_{0}^{+}$increases as the energy $E_{i}$ decreases from 16.6 to 8.2 to $4 \mathrm{meV}$. This is in qualitative agreement with the experiments [7], where the SH mode was nearly undetectable at $16.6 \mathrm{meV}$.

Figure 3 shows a comparison of the SC, NSC (Appendix) and experimental data for the specular $\mathrm{SH}$ inelastic strength at $8.2 \mathrm{meV}$ and scattering plane near the $\Gamma \mathrm{M}$ azimuth. The strong SH signal at small $|\mathbf{Q}|$ is present in both the SC and NSC results.

In the experiments [7], $\mathrm{QI}_{0}^{+}$of the $\mathrm{SH}$ branch at small $|\mathbf{Q}|$ was larger than that of the LA branch. At $E_{i}=8.2 \mathrm{meV}$ and small $|\mathbf{Q}|$, the SH signal was significantly more intense for a scattering plane at $2.6^{\circ}$ to the $Г \mathrm{M}$ azimuth of the xenon than for the plane at $2.6^{\circ}$ to the $\Gamma \mathrm{K}$ azimuth. $Q I_{0}^{+}$

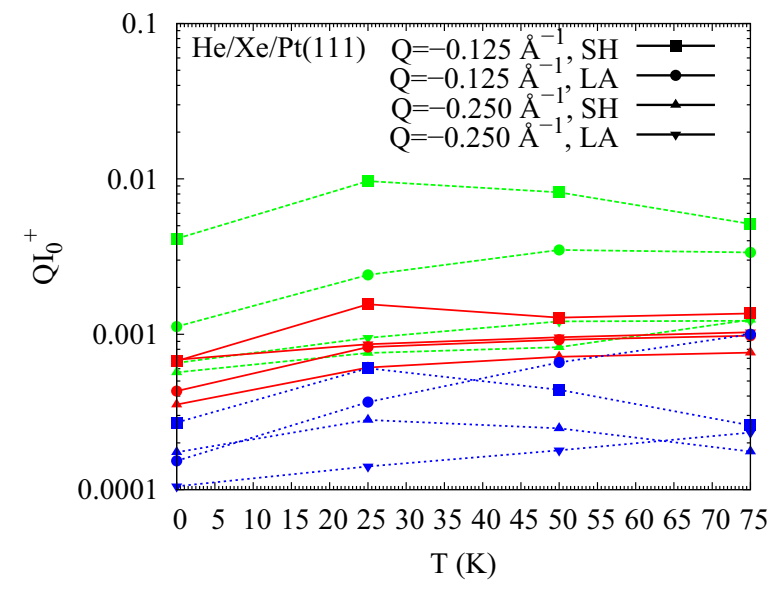

FIG. 2. Specular inelastic 1-phonon creation strengths $Q I_{0}^{+}$as a function of temperature $T$ for the cases treated in Fig. 1. $Q I_{0}^{+}$is plotted on a logarithmic scale. Identifications as in Fig. 1.

decreased strongly with increasing $|\mathbf{Q}|$ and the ratio of $Q I_{0}^{+}$ strengths (" $\Gamma \mathrm{M} / \Gamma \mathrm{\Gamma K}$ ") was $\approx 4$ at $|\mathbf{Q}| \approx 0.1 \AA^{-1}$ and $\approx 2$ at $|\mathbf{Q}| \approx 0.2 \AA^{-1}$. It was difficult at small $|\mathbf{Q}|$ to separate the inelastic strength of the LA branch from that of a substrate excitation in the experiments, but it was suggested [7], based on calculations of other workers, that the excitation probability of the LA branch would vary slowly with $|\mathbf{Q}|$.

Many of these features are reproduced by the calculations. For a given $\mathrm{SH}$ mode $\mathrm{QI}_{0}^{+}$increases as $E_{i}$ decreases from 16.6 to 8.2 to $4 \mathrm{meV}$. At small $|\mathbf{Q}|$ there is quite a range of conditions where the $\mathrm{SH}$ branch $Q I_{0}^{+}$is larger than that of the LA branch. The excitation probability of the LA branch varies slowly with $|\mathbf{Q}|$ at $E_{i}=8.2 \mathrm{meV}$, but not at $4 \mathrm{meV}$ nor at $16.6 \mathrm{meV}$.

Table I gives more data on the calculated specular inelastic intensities of the LA and SH branches, including the conditions of the most extensive experimental data [7], $E_{i}=8.2 \mathrm{meV}$

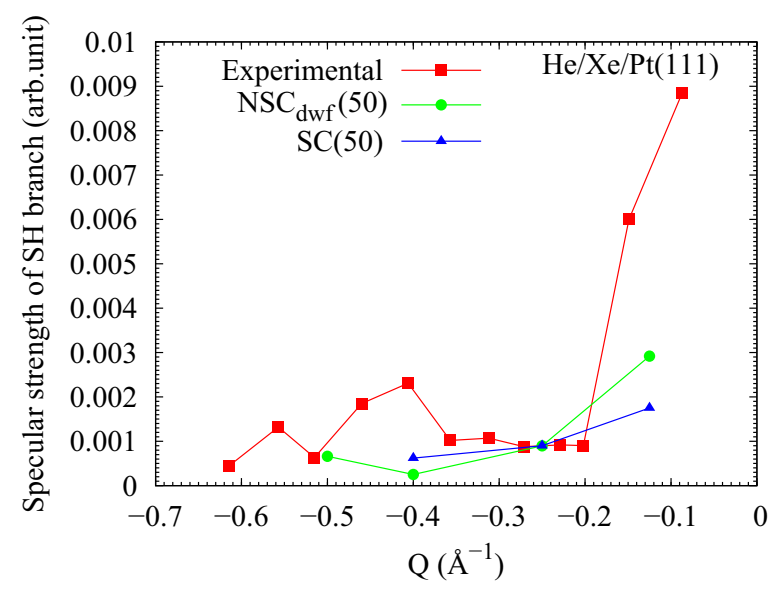

FIG. 3. Specular inelastic strength $\left(Q I_{0}^{+}\right)$of the $\mathrm{SH}$ branch of $\mathrm{Xe} / \mathrm{Pt}(111)$ for $E_{i}=8.2 \mathrm{meV}$ and scattering plane at $2.6^{\circ}$ to the monolayer $\Gamma \mathrm{M}$ azimuth $\left(L_{n n}=4.33 \AA, T=50 \mathrm{~K}\right)$. The results of the SC and NSC approximations (including the phase space factor $k_{f} / k_{i z}$ ) are compared to data from the HAS experiment. [7] The three sets of data have been scaled to agree at $\mathbf{Q}=-0.25 \AA^{-1}$. 
TABLE I. Specular inelastic strengths $Q I_{0}^{+}$by SC and NSC calculations. ${ }^{4} \mathrm{He}$ atoms of energy $E_{i}$ (in meV) are incident on monolayer $\mathrm{Xe} / \mathrm{Pt}(111)$ with nearest-neighbor distance $L_{n n}=4.33 \AA$ and scan-curve conditions $\left(\theta_{\mathrm{SD}}=95.8^{\circ}\right)$, creating phonons at wave vectors $\mathbf{Q}$ (in $\AA^{-1}$ ) and polarizations and scattering planes as noted; the angle of incidence $\theta_{i}$ varies with the scattering case but not with the temperature. $\mathrm{SC}(T)$ values are calculated with $N_{\mathbf{Q}}=15$. NSC values have an arbitrary but internally consistent normalization. NSC $(T)$ is evaluated using Eq. (A1).

\begin{tabular}{|c|c|c|c|c|c|c|c|}
\hline \multirow[b]{2}{*}{$E_{i}$} & \multirow{2}{*}{$\begin{array}{c}\text { Polarization } \\
\text { Q }\end{array}$} & \multicolumn{2}{|c|}{ SH } & \multicolumn{2}{|c|}{ LA } & \multicolumn{2}{|c|}{ LA/SH } \\
\hline & & -0.125 & -0.25 & -0.125 & -0.25 & -0.125 & -0.25 \\
\hline \multirow[t]{4}{*}{$4^{\mathrm{a}}$} & $\mathrm{NSC}(0)$ & 0.367 & $6.5 \times 10^{-2}$ & $7.9 \times 10^{-2}$ & $3.3 \times 10^{-2}$ & 0.22 & 0.51 \\
\hline & $\mathrm{SC}(0)$ & $4.1 \times 10^{-3}$ & $5.4 \times 10^{-4}$ & $1.1 \times 10^{-3}$ & $6.4 \times 10^{-4}$ & 0.27 & 1.2 \\
\hline & $\mathrm{NSC}(50)$ & 1.81 & 0.175 & 0.233 & $5.6 \times 10^{-2}$ & 0.13 & 0.32 \\
\hline & $\mathrm{SC}(50)$ & $8.3 \times 10^{-3}$ & $9.6 \times 10^{-4}$ & $3.7 \times 10^{-3}$ & $1.2 \times 10^{-3}$ & 0.45 & 1.25 \\
\hline \multirow[t]{4}{*}{$8.2^{\mathrm{a}}$} & $\mathrm{NSC}(0)$ & $6.33 \times 10^{-2}$ & $3.51 \times 10^{-2}$ & $3.42 \times 10^{-2}$ & $5.66 \times 10^{-2}$ & 0.54 & 1.61 \\
\hline & $\mathrm{SC}(0)$ & $6.7 \times 10^{-4}$ & $3.6 \times 10^{-4}$ & $4.3 \times 10^{-4}$ & $6.8 \times 10^{-4}$ & 0.64 & 1.89 \\
\hline & $\mathrm{NSC}(50)$ & 0.280 & $9.40 \times 10^{-2}$ & $9.10 \times 10^{-2}$ & $8.75 \times 10^{-2}$ & 0.32 & 1.03 \\
\hline & $\mathrm{SC}(50)$ & $1.3 \times 10^{-3}$ & $7.2 \times 10^{-4}$ & $9.2 \times 10^{-4}$ & $9.6 \times 10^{-4}$ & 0.71 & 1.33 \\
\hline \multirow[t]{4}{*}{$8.2^{\mathrm{b}}$} & $\mathrm{NSC}(0)$ & $3.58 \times 10^{-2}$ & $9.84 \times 10^{-3}$ & $8.29 \times 10^{-3}$ & $8.80 \times 10^{-3}$ & 0.23 & 0.89 \\
\hline & $\mathrm{SC}(0)$ & $3.8 \times 10^{-4}$ & $7.2 \times 10^{-5}$ & $6.9 \times 10^{-5}$ & $1.1 \times 10^{-4}$ & 0.18 & 1.53 \\
\hline & $\mathrm{NSC}(50)$ & 0.161 & $2.34 \times 10^{-2}$ & $2.31 \times 10^{-2}$ & $1.37 \times 10^{-2}$ & 0.14 & 0.58 \\
\hline & $\mathrm{SC}(50)$ & $1.1 \times 10^{-3}$ & $8.9 \times 10^{-5}$ & $2.0 \times 10^{-4}$ & $1.9 \times 10^{-4}$ & 0.18 & 2.1 \\
\hline
\end{tabular}

$\overline{\bar{a}} \mathbf{Q}$ and scattering plane at $2.6^{\circ}$ relative to the $\Gamma \mathrm{M}$ azimuth. The phonon energies are $0.64,1.24,1.12$, and 2.18 meV, respectively. The corresponding values of $\theta_{i}$ are: (i) at $E_{i}=4 \mathrm{meV}, 43.2^{\circ}, 37.8^{\circ}, 40.7^{\circ}$, and $31.3^{\circ}$; (ii) at $E_{i}=8.2 \mathrm{meV}, 45.3^{\circ}, 42.5^{\circ}, 44.2^{\circ}$, and $40.2^{\circ}$.

${ }^{\mathrm{b}} \mathbf{Q}$ and scattering plane at $2.6^{\circ}$ relative to the $\Gamma \mathrm{K}$ azimuth. The phonon energies are $0.64,1.27,1.11$, and $2.16 \mathrm{meV}$, respectively. The corresponding $\theta_{i}$ are: $45.3^{\circ}, 42.4^{\circ}, 44.2^{\circ}$, and $40.2^{\circ}$.

and $T=50 \mathrm{~K}$. The excitation probability of the LA branch is definitely smaller than that of the SH branch for $|\mathbf{Q}|=$ $0.125 \AA^{-1}$ at these $E_{i}$. At $8.2 \mathrm{meV}, Q I_{0}^{+}(\mathrm{SH})$ decreases on going from $\mathbf{Q}=-0.125 \AA^{-1}$ to $\mathbf{Q}=-0.25 \AA^{-1}$ along both azimuths and the strength is greater near the $\Gamma \mathrm{M}$ azimuth. The ratio of the value of $Q I_{0}^{+}(\mathrm{SH})$ near the $\Gamma \mathrm{M}$ azimuth to that near the $\Gamma \mathrm{K}$ azimuth increases on going from $\mathbf{Q}=-0.125 \AA^{-1}$ to $\mathbf{Q}=-0.25 \AA^{-1}$, counter to the experimental result. The ratio of $Q I_{0}^{+}$for the LA phonon to that of the SH phonon is larger in the SC data than the NSC data.

The most significant element in the comparison of the modeling and the $\mathrm{Xe} / \mathrm{Pt}(111)$ experiment is [7] that the frequency spectra of both the experimental SH and LA branches are reproduced very well by the interaction model, which is primarily that of the gas phase Xe-Xe pair potential [51]. The fact that our calculations of excitation probabilities reproduce trends for the dispersive branches in the experimental data supports our identification of the Novaco-McTague rotational alignment [59] as the process that enables excitation of the $\mathrm{SH}$ branch in these experiments. The crucial role of alignment in the inelastic scattering was shown also for a xenon multilayer [60]. We cannot exclude a contribution of extrinsic terms (e.g., defects) to the observed symmetrybreaking intensity and a very useful measurement would be the absolute strength of the SH excitation.

\section{B. $\mathrm{Xe} / \mathrm{Cu}(001)$}

As a preliminary we note two experiments for $\mathrm{He} / \mathrm{Xe} / \mathrm{Cu}(110)$ with $E_{i} \simeq 18.2 \mathrm{meV}$ that show strong inelastic effects. Near $75 \mathrm{~K}$, the specular reflectivity $\left(\theta_{i}=45^{\circ}\right)$ of the monolayer was [61] less than $1 \%$, while in another experiment [62], the diffracted intensities increased by a factor of 3 to 4 as the temperature decreased from 55 to $25 \mathrm{~K}$. Although the higher-order-commensurate monolayers of $\mathrm{Xe} / \mathrm{Cu}(110)$ differ [9] from the incommensurate $\mathrm{Xe} / \mathrm{Pt}(111)$, these values are comparable to estimates using the data in Fig. 1 for $\theta_{i} \simeq 45^{\circ}$ and $E_{i}=16.6 \mathrm{meV}, N_{0}=0.002-0.003$ at $75 \mathrm{~K}$ and $N_{0}(25) / N_{0}(50)=3.3-3.6$.

For $\mathrm{Xe} / \mathrm{Cu}(001)$, Graham et al. [8] measured a dispersion curve at $50 \mathrm{~K}$ of an incommensurate monolayer. Their experiment had $E_{i} \simeq 8 \mathrm{meV}$ and included temperatures in the range $45-75 \mathrm{~K}$; the scattering plane was along the [100] azimuth of the $\mathrm{Cu}(001)$. They interpreted the lattice constant data derived from diffraction measurements along this azimuth as showing a phase transition in a triangular monolayer solid at $T \approx 65-70 \mathrm{~K}$. The strength $Q I_{0}^{+}$of the specular inelastic peak for an excitation with $\mathbf{Q} \approx-0.2 \AA^{-1}$ along [100] increased [63] by a factor of 10 as $T$ decreased from 75 to $45 \mathrm{~K}$. On the basis of a symmetry argument that the intensity of the SH branch would be zero or exceedingly small in this geometry, they assigned the dispersion curve as an LA branch. However, a fit to the data then required anomalously small $\mathrm{Xe}-\mathrm{Xe}$ force constants and there is no candidate structural transition to match the proposed xenon phase transition.

If there is a symmetry-breaking mechanism, the observed dispersion curve can be assigned [2] to an SH branch with "normal" force constants. Our SC calculations show that a $1^{\circ}$ misalignment of the Xe $\Gamma \mathrm{M}$ azimuth relative to the $\mathrm{Cu}$ [100] azimuth enables excitation of the SH phonon with $|\mathbf{Q}|=-0.2 \AA^{-1}$ for $E_{i}=8.1 \mathrm{meV}$. However the temperature dependence of $Q I_{0}^{+}(T)$ is very different than reported [63]. Comparing results for a triangular lattice with $L_{n n}=4.41 \AA$ (the experimental result at $50 \mathrm{~K}$ ) and $\theta_{i}=43.8^{\circ}$, the ratio $Q I_{0}^{+}(50) / Q I_{0}^{+}(75)$ is 1.3 while if the comparison is for lattices with $L_{n n}=4.41 \AA$ at $50 \mathrm{~K}$ and $L_{n n}=4.52 \AA$ and $\theta_{i}=44.2^{\circ}$ at $75 \mathrm{~K}$ [64] (both experimental $L_{n n}$-values) the ratio is 4.4. These values are considerably less than the experimental ratio 
of 10 . Further, the ratio of the (01) to the (00) diffraction peak intensity at $50 \mathrm{~K}$ is $0.3-0.4$, which is considerably larger than the experimental estimate [63] of less than 0.01 at 50-70 K.

We propose that the differences between the experiment and modeling can be understood in terms of rotation of the xenon lattice relative to the substrate axes. In fact there is [8] a two-dimensional (2D) diffraction pattern for the $\mathrm{Xe} / \mathrm{Cu}(001)$ at $70 \mathrm{~K}$ that shows pairs of domains rotated by a few degrees in both directions from the [100] azimuth. This creates a symmetry-breaking mechanism for the $\mathrm{SH}$ branch. The diffraction measurement along the [100] azimuth can be rationalized as probing the shoulder of the Xe diffraction peak and the phase transition might be a thermal activation of rotation of the monolayer. Temperature dependence of the monolayer alignment should be observable in an experiment that makes systematic $2 \mathrm{D}$ scans of the monolayer scattering.

\section{Xe/graphite}

Bracco et al. [16] measured the diffraction of ${ }^{4} \mathrm{He}$ atoms of energy $E_{i}=63.77 \mathrm{meV}$ at normal incidence on a commensurate xenon monolayer on graphite at $17 \mathrm{~K}$. They determined the probabilities for the specular elastic scattering and 15 shells of diffraction peaks with reciprocal lattice vectors of magnitude $|\mathbf{G}| \leqslant 6 G_{0}$, where $G_{0} \simeq 1.70 \AA^{-1}$ is the magnitude of the primitive reciprocal lattice vector. Their total elastic scattering probability is $N_{\mathrm{E}}=0.112$. The energy resolution was rather limited [65], with FWHM $=9.6 \mathrm{meV}$ and a speed ratio of about 22.

Our SC calculations at $17 \mathrm{~K}$ give $N_{\mathrm{E}}[\mathrm{SC}]=0.071$. The difference from the experimental data arises mostly for large angle scattering (large $|\mathbf{G}|$ ). The calculated total of the elastic scattering through the tenth shell $\left(\sqrt{21} G_{0}\right)$ is 0.063 and it is 0.073 in the experimental data. A plot of the diffraction intensities $I(\mathbf{G})$ is shown in Fig. 4. The $\ln I$ (for a given channel, not multiplied by the degeneracy) are shown for the SC calculation (diamonds), the experiment ("Bracco," dots), and the NSC approximation (squares) constructed from the intensities calculated by Hutson and Schwartz [24] for a static lattice.

Hutson and Schwartz [24] obtained the static lattice diffraction strengths $I(\mathbf{G}$, static $)$ with a close-coupling calculation for the HFD-1 He-Xe potential. They then made the Debye-Waller correction given in Eq. (A2) without the $G^{2}$ term and got diffraction strengths that sum to $N_{\mathrm{E}}=0.117$, close to the experimental sum. We have adjusted their data to include the lateral vibrations using Eq. (A2) and the mean-square displacement evaluated for the Xe/graphite model stated in Sec. IV,$\left\langle u_{x}^{2}\right\rangle=\left\langle u_{y}^{2}\right\rangle=0.0075 \AA^{2}$. The final results are shown as the squares in Fig. 4 and give $N_{\mathrm{E}}[\mathrm{NSC}]=0.075$, close to the $\mathrm{SC}$ sum. With the exception of the channel with $G / G_{0}=5$, the channels with strong diffraction are obtained in both calculations.

The most puzzling discrepancy between the SC calculation and the experiment is for the (50) diffraction peak, at $G / G_{0}=$ 5 in Fig. 4. Some artifacts at large scattering angles, such as scattering from background gas and inelastic scattering, might blur minima, but the data show a strong (50) peak. The puzzle is that Bracco et al. fit this strong peak as part of the overall fit with a corrugated hard wall and Hutson and Schwartz [24] reproduce the peak with what we classify

\section{Commensurate $\mathrm{Xe} /$ graphite perpendicular incidence diffraction}

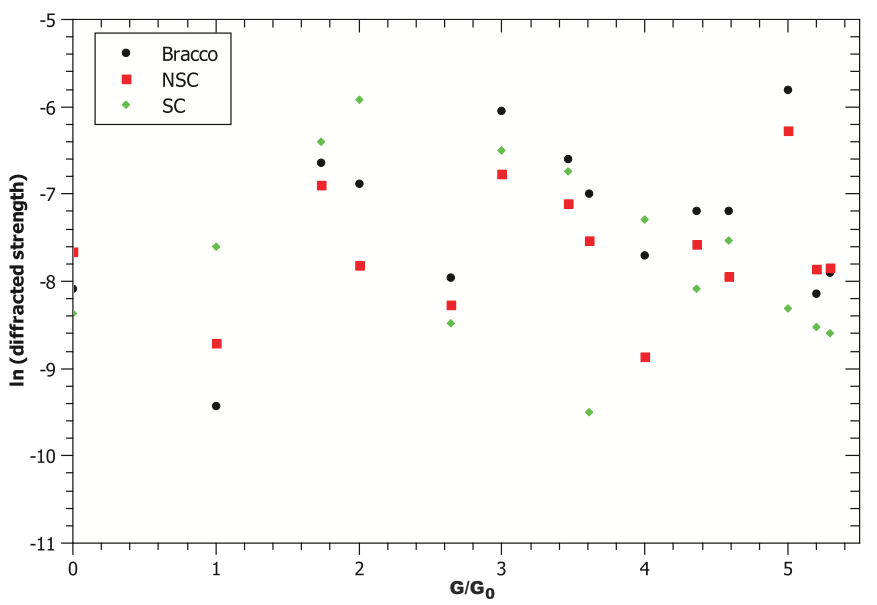

FIG. 4. The natural logarithm of the diffraction strength in a channel $\ln I$ with the given $G / G_{0}$ is shown for scattering of $63.77 \mathrm{meV} \mathrm{He}$ atoms at perpendicular incidence on a commensurate monolayer of xenon on graphite at $17 \mathrm{~K}$. The dots show the experimental data of Bracco et al. [16], the diamonds are the results of our SC calculations, and the squares (NSC) are the results of a scaling of the Hutson and Schwartz [24] calculation to include the effect of lateral vibrations in the monolayer, using Eq. (A2). This presentation is a composite of diffraction peaks for six azimuths.

an NSC approximation, while our SC calculation, with a nominally more realistic treatment of the large inelastic attenuation, misses it.

Hutson and Schwartz assign the (50) peak as a rainbow peak. In fact, after freezing out the inelastic scattering by using $\hbar \omega_{0 \|}=\hbar \omega_{\perp}=70 \mathrm{meV}$ in the lattice dynamics we reproduce the static lattice results of Hutson and Schwartz. That is, the strong inelastic scattering suppresses the quantum rainbow, a possibility noted by Garibaldi et al. [66]. This leaves open the question of what must be added to the model to lead to a strong (50) peak.

There still is a question of what effect the dispersion in $\omega_{\perp}$ arising from the mixing of the $\mathrm{S}$ mode and the substrate Rayleigh wave [52] has. Since the S mode contributes $75 \%$ of the diffuse inelastic strength in our SC calculation, this effect could lead to changes in the theory side of the comparison with the Bracco experiment. There certainly is a need for a more systematic study of Xe/graphite because so much is already known for it that one expects a constructive confrontation of the modeling and experiment.

\section{CONCLUSIONS}

The implementation of the SC theory of time-dependent elastic and inelastic He-atom scattering from a monolayer solid film, including diffuse inelastic scattering by the phonon modes, is very stable numerically. The total norm of the wave function is conserved to better than $0.01 \%$ throughout the propagation and independent of the number of coupled channels, e.g., 4300, 5300, and 8700 in the finite temperature calculations at $E_{i}=4,8.2$, and $16.6 \mathrm{meV}$, respectively.

The results for $\mathrm{He} / \mathrm{Xe} / \mathrm{Pt}(111)$ at $E_{i}=4-16 \mathrm{meV}$, with large strengths in nonspecular channels for both elastic and 
inelastic scattering, illustrate how corrugated the target is and emphasize the degree to which a treatment of the inelastic scattering without the diffraction is an extreme simplification. This concern may have relevance to future phonon spectroscopy on a system such as $\mathrm{He} / \mathrm{Sb}(111)$, where HAS experiments have been used [67] to infer a hard-wall corrugation that is comparable to those [37,53] for $\mathrm{He} / \mathrm{Xe} / \mathrm{Pt}(111)$ and $\mathrm{He} / \mathrm{Xe} /$ graphite.

The SC theory includes effects of inelastic scattering both on the elastic channel strengths and on the distribution of inelastic channel strengths. These effects, the Debye-Waller attenuations, are larger than in previous estimates using (NSC) perturbation approximations [24,38]. The xenon monolayers are relatively soft and the $\mathrm{He}$ atom couples strongly to the monolayer modes. The SC calculations show that the inelastic scattering is large and that a perturbation approximation is only qualitatively useful.

The calculations for $\mathrm{He} / \mathrm{Xe} / \mathrm{Pt}(111)$ reported in the Supplemental Material [17] show an anomalous increase in the total elastic strength $N_{\mathrm{E}}$ for $E_{i}=16.6 \mathrm{meV}$ at $75 \mathrm{~K}$ that is accompanied by an increase in diffracted strength in nonspecular channels and a reduction in the diffuse scattering by S-branch phonons. A similar anomaly occurs in other examples with strong S-branch scattering [68].

The total elastic scattering probability $N_{\mathrm{E}}$ for $E_{i}=$ $16.6 \mathrm{meV}$ is about 0.5 at $0 \mathrm{~K}$ and $0.07-0.10$ at $50 \mathrm{~K}$. These values are larger than the estimated probability of less than 0.05 for the $200 \mathrm{eV}$ electrons in a typical LEED experiment [48]. The LEED electrons have a high enough speed that approximating thermal effects by a randomizing term in LEED intensity calculations [47] leads to fitted S-mode energies $\omega_{\perp}$ that agree with direct HAS measurements [69].

Our work shows the importance of having structural (diffraction) measurements that accompany the inelastic scattering data. The $\mathrm{SC}$ results for $\mathrm{He} / \mathrm{Xe} / \mathrm{Cu}(001)$ diffraction and inelastic strengths differ from the inferences based on the experiment $[4,8]$. However, the differences can be reconciled using a Xe monolayer alignment that is consistent with the experiment. The $\mathrm{SC}$ calculations for $\mathrm{He} / \mathrm{Xe} / \mathrm{Pt}(111)$ inelastic scattering are in general agreement with the experimental data [7] when the experimental monolayer alignment is used.

The poor agreement with the channel strengths in the Xe/graphite diffraction experiment and for the trend of the symmetry breaking SH strength with wave number in the $\mathrm{Xe} / \mathrm{Pt}(111)$ experiment is surprising in light of previous calculations [5,24] that gave better agreement using more primitive approximations for the inelastic scattering. It has been noted [37] that a satisfactory agreement for Xe/graphite diffraction intensities was obtained in spite of the omission of several effects from the calculations. We suggest that previous agreement depended on a fortuitous cancellation of errors that separating the strong coupling effects in the scattering theory removes. Because there is so much evidence supporting the interaction models in these examples, part of the problem may arise from the idealizations of the state of the monolayer target. This could be tested with more systematic annealing of the target and with experiments at lower beam energies where it is feasible to make a much finer sampling of the Brillouin zone for evaluating the diffuse scattering in the theory. These questions are basic to the quantitative analysis of intensities in low energy helium scattering.
We believe the SC formulation gives a viable theory of the strong inelastic scattering at the beam energies used for measurements of dispersive phonon branches in physically adsorbed monolayers. Treating the quantized lattice dynamics of the target is an essential step for modeling low energy helium scattering. The results show the complexity arising from the strong coupling; there are competing effects of excitation of the $\mathrm{S}$ and the SH and LA branches. Much is known about the ingredients for a quantitative theory of the low energy scattering, but detailed tests of the theory will require difficult systematic experiments.

\section{APPENDIX: NSC APPROXIMATION}

In previous work $[5,38]$ we used a non-self-consistent (NSC) version of Eqs. (13)-(15) as a simpler approximation to the scattering theory. The last two $\left(C_{\lambda}\right.$-coupling $)$ terms in Eq. (13) were neglected. No phonon annihilation processes were included, so Eq. (15) did not enter. Phonon creation was limited to a specific mode and there was no provision for diffuse inelastic scattering. The total norm of the elastic wave function $\psi_{0}$ was constant because the coupling terms in Eq. (13) were omitted. The calculated elastic and inelastic strengths were multiplied by Debye-Waller attenuation factors and the inelastic strengths also by the factor $[1+\bar{n}(\mathbf{q})]$ to get estimates (albeit with arbitrary normalizations) to compare with experimental data for the inelastic strengths at finite temperature. These drastic approximations simplified the calculations and were sufficient for the primary goal of that work [5], which was to demonstrate a symmetry-breaking mechanism that leads to excitation of $\mathrm{SH}$ modes near high-symmetry directions in HAS experiments. The main features and trends in a comprehensive body of experimental HAS data [7] for monolayers of $\mathrm{Xe}, \mathrm{Kr}$, and $\mathrm{Ar}$ on $\mathrm{Pt}(111)$ were reproduced.

To be explicit, the effect of monolayer vibrations on elastic and energy loss strengths in the NSC approximation $[5,38]$ is estimated using the harmonic approximation to the DebyeWaller exponent [70] $W_{\mathrm{DW}}(T)$. The final calculated elastic strength is multiplied by $\exp \left[-2 W_{\mathrm{DW}}(T)\right]$, which reduces it, while the phonon creation strengths are multiplied by the factor

$$
f_{\mathbf{q}}(T)=\left[1+\bar{n}_{\mathbf{q}}\right] \exp \left[-2 W_{\mathrm{DW}}(T)\right],
$$

to allow for excitation from a thermal equilibrium phonon population. [The corresponding NSC phonon annihilation factor has $\bar{n}_{\mathbf{q}}$ in place of $1+\bar{n}_{\mathbf{q}}$ in Eq. (A1).] $f_{\mathbf{q}}$ is a product of two factors: $\left[1+\bar{n}_{\mathbf{q}}\right]$, which increases, and the exponential, which decreases with increasing temperature. Hence, depending on their relative weights, the phonon creation strengths may either increase or decrease with temperature. This method of including the thermal motion of the target was developed for the weak-coupling regime of inelastic thermal neutron scattering [23]. The thermal effects represented by the factors in $f_{\mathbf{q}}$ are included implicitly in the SC theory.

The NSC approximation to the diffracted intensities for a commensurate lattice is constructed from the intensities calculated for a static lattice $I(\mathbf{G}$, static $)$ as follows [71]:

$$
\begin{aligned}
& I(\mathbf{G}, \text { NSC }) \\
& \quad=I(\mathbf{G}, \text { static }) \times \exp \left[-\left(\Delta^{\prime} \mathrm{k}_{\mathrm{z}}\right)^{2}\left\langle\mathrm{u}_{\mathrm{z}}^{2}\right\rangle-\left(\mathrm{G}^{2} / 2\right)\left\langle\mathrm{u}_{\mathrm{x}}^{2}+\mathrm{u}_{\mathrm{y}}^{2}\right\rangle\right] .
\end{aligned}
$$


Equation (A2) is used in a discussion of a previous closecoupling calculation [24] in Sec. VC. The $\Delta^{\prime} k_{z}$ denotes the change in the $\mathrm{z}$ component of the wave vector with the Beeby correction included and $\left\langle u_{\alpha}^{2}\right\rangle$ denote the components of mean-square displacement of a monolayer atom (nonsingular for a commensurate lattice). Note that the exponential dependence on $\left\langle u_{z}^{2}\right\rangle$ in Eq. (A2) makes the NSC calculations for $\mathrm{He} / \mathrm{Xe} /$ graphite depend sensitively on the value of $\omega_{\perp}$ for the large $\Delta^{\prime} k_{z}$ in the Bracco et al. experiment [55].
[1] A. P. Graham, Surf. Sci. Rep. 49, 115 (2003).

[2] L. W. Bruch, R. D. Diehl, and J. A. Venables, Rev. Mod. Phys. 79, 1381 (2007), and references therein.

[3] B. Gumhalter, Phys. Rep. 351, 1 (2001).

[4] A. Šiber, B. Gumhalter, J. Braun, A. P. Graham, M. Bertino, J. P. Toennies, D. Fuhrmann, and C. Wöll, Phys. Rev. B 59, 5898 (1999).

[5] L. W. Bruch and F. Y. Hansen, J. Chem. Phys. 122, 114714 (2005); The present paper uses lower case boldface to denote $3 \mathrm{D}$ vectors and upper case boldface to denote $2 \mathrm{D}$ vectors such as the monolayer reciprocal lattice vectors $\mathbf{G}$ and mode wave vectors $\mathbf{Q}$.

[6] B. F. Mason and B. R. Williams, Surf. Sci. 148, L686 (1984), $\left(E_{i} \approx 20 \mathrm{meV}\right)$.

[7] L. W. Bruch, A. P. Graham, and J. P. Toennies, J. Chem. Phys. 112, 3314 (2000).

[8] A. P. Graham, M. F. Bertino, F. Hofmann, J. P. Toennies, and C. Wöll, J. Chem. Phys. 106, 6194 (1997); 107, 4445 (1997); A. P. Graham and M. F. Bertino (private communications).

[9] C. Boas, M. Kunat, U. Burghaus, B. Gumhalter, and C. Wöll, Phys. Rev. B 68, 075403 (2003).

[10] B. S. Zhao, G. Meijer, and W. Schöllkopf, Phys. Rev. Lett. 104, 240404 (2010); W. Zhang, J. H. Lee, H. A. Kim, B. G. Jin, B. J. Kim, L. Y. Kim, B. S. Zhao, and W. Schöllkopf, Chem. Phys. Chem. 17, 3670 (2016).

[11] B. A. J. Lechner, H. Hedgeland, W. Allison, J. Ellis, and A. P. Jardine, Rev. Sci. Instrum. 84, 026105 (2013).

[12] E.g., A. Tamtögl, E. Bahn, J. Zhu, P. Fouquet, J. Ellis, and W. Allison, J. Phys. Chem. C 119, 25983 (2015).

[13] H. Shichibe, Y. Satake, K. Watanabe, A. Kinjyo, A. Kunihara, Y. Yamada, M. Sasaki, W. W. Hayes, and J. R. Manson, Phys. Rev. B 91, 155403 (2015).

[14] P. Kraus, A. Tamtögl, M. Mayrhofer-Reinhartshuber, F. Apolloner, C. Gösweiner, S. Miret-Artés, and W. E. Ernst, J. Phys. Chem. C 119, 17233 (2015).

[15] M. del Cueto, A. S. Muzas, G. Füchsel, F. Gatti, F. Martin, and C. Díaz, Phys. Rev. B 93, 060301(R) (2016).

[16] G. Bracco, P. Cantini, A. Glachant, and R. Tatarek, Surf. Sci. 125, L81 (1983).

[17] See Supplemental Material at http://link.aps.org/supplemental/ 10.1103/PhysRevB.95.214303 for a discussion of the role of detailed balance relations for the energy gain and energy loss inelastic scattering results for $\mathrm{Xe} / \mathrm{Pt}(111)$, a treatment of the zero temperature limit for diffraction with a beam at perpendicular incidence, and seven supplementary figures. There also are sections describing a test of sensitivity to choice of $N_{\mathbf{Q}}$, the evidence supporting neglect of multiphonon processes here, analysis of trapping phenomena, and further comparisons of the results of the SC and NSC calculations.

[18] J. A. Barker and D. J. Auerbach, Surf. Sci. Rep. 4, 1 (1985).

[19] A. S. Sanz and S. Miret-Artés, Phys. Rep. 451, 37 (2007).

[20] A. C. Levi and H. Suhl, Surf. Sci. 88, 221 (1979).
[21] S. Daon and E. Pollak, J. Chem. Phys. 142, 174102 (2015), and references therein.

[22] S. Daon, E. Pollak, and S. Miret-Artés, J. Chem. Phys. 137, 201103 (2012).

[23] W. Marshall and S. W. Lovesey, Theory of Thermal Neutron Scattering (Oxford University Press, Oxford, 1971).

[24] J. M. Hutson and C. Schwartz, J. Chem. Phys. 79, 5179 (1983).

[25] H. Jónsson, J. H. Weare, T. H. Ellis, G. Scoles, and U. Valbusa, Phys. Rev. B 30, 4203 (1984).

[26] K. D. Gibson, C. Cerjan, J. C. Light, and S. J. Sibener, J. Chem. Phys. 88, 7911 (1988).

[27] R. A. Aziz, U. Buck, H. Jónsson, J.-C. Ruiz-Suárez, B. Schmidt, G. Scoles, M. J. Slaman, and J. Xiu, J. Chem. Phys. 91, 6477 (1989); 93, 4492(E) (1990); The HFD-B2 model incorporates improved values for the dispersion energy coefficients $C_{6}, C_{8}, C_{10}$ in the HFD-1 model [24].

[28] V. Celli and A. A. Maradudin, Phys. Rev. B 31, 825 (1985); W. Brenig, Z. Phys. B 36, 227 (1980); see also discussion at Eq. (265) of Ref. [3].

[29] M. D. Stiles, J. W. Wilkins, and M. Persson, Phys. Rev. B 34, 4490 (1986); B. Jackson, J. Chem. Phys. 88, 1383 (1988).

[30] S. Miret-Artés, Surf. Sci. 339, 205 (1995).

[31] In implementing the coupling to the diffuse background, Kraus et al. [14] lump the couplings into a single $O(1)$ coupling to an active phonon, in contrast to our $3 N_{\mathbf{Q}} O\left(1 / \sqrt{N_{\mathbf{Q}}}\right)$ couplings of wave function amplitudes, Eqs. (13)-(15), and then average the calculated intensities over a Debye spectrum of phonon frequencies.

[32] A. Šiber and B. Gumhalter, Phys. Rev. B 71, 081401 (2005).

[33] D. Eichenauer, U. Harten, J. P. Toennies, and V. Celli, J. Chem. Phys. 86, 3693 (1987).

[34] K. D. Gibson and S. J. Sibener, J. Chem. Phys. 88, 7862 (1988).

[35] R. Kosloff and C. Cerjan, J. Chem. Phys. 90, 7556 (1989).

[36] E. R. Bittner and J. C. Light, J. Chem. Phys. 99, 8229 (1993).

[37] A. Šiber and B. Gumhalter, Surf. Sci. 529, L269 (2003); Prog. Surf. Sci. 74, 375 (2003).

[38] F. Y. Hansen and L. W. Bruch, J. Chem. Phys. 127, 204708 (2007).

[39] J. R. Manson and V. Celli, Phys. Rev. B 39, 3605 (1989).

[40] For a recent quantum treatment, see B. Gumhalter, J. Phys.: Condens. Matter 24, 104015 (2012).

[41] The linear coupling does lead to significant multiphonon excitations, see A. Šiber and B. Gumhalter, Phys. Rev. Lett. 90, 126103 (2003).

[42] L. W. Bruch, M. W. Cole, and E. Zaremba, Physical Adsorption: Forces and Phenomena (Oxford University Press, Oxford, 1997).

[43] M. Nest and R. Kosloff, J. Chem. Phys. 127, 134711 (2007); U. Lorenz and P. Saalfrank, ibid. 140, 044106 (2014).

[44] H.-D. Meyer and G. A. Worth, Theor. Chem. Acc. 109, 251 (2003).

[45] Y.-W. Lin and G. Wolken, Jr., J. Chem. Phys. 65, 2634 (1976). 
[46] Z. Medina and B. Jackson, J. Chem. Phys. 125, 224703 (2006); B. Lepetit, D. Lemoine, Z. Medina, and B. Jackson, ibid. 134, 114705 (2011).

[47] M. A. Van Hove and S. Y. Tong, Surface Crystallography by LEED: Theory, Computation, and Structural Results (Springer, New York, 1979).

[48] J. B. Pendry, Low Energy Electron Diffraction: The Theory and its Applications to Determination of Surface Structure (Academic Press, London, 1974).

[49] R. D. Brown, Q. Tong, J. S. Becker, M. A. Freedman, N. A. Yufa, and S. J. Sibener, Faraday Discuss. 157, 307 (2012).

[50] M. Li, J. R. Manson, and A. P. Graham, Phys. Rev. B 65, 195404 (2002).

[51] A. K. Dham, W. J. Meath, A. R. Allnatt, R. A. Aziz, and M. J. Slaman, Chem. Phys. 142, 173 (1990).

[52] J. P. Toennies and R. Vollmer, Phys. Rev. B 40, 3495 (1989).

[53] L. W. Bruch, F. Y. Hansen, and F. Traeger, J. Chem. Phys. 134, 194308 (2011).

[54] L. W. Bruch and A. D. Novaco, Phys. Rev. B 77, 125435 (2008).

[55] A wide range of values is quoted for the $\mathrm{S}$ mode of Xe/graphite. Other values are $3.2 \mathrm{meV}$ from Toennies and Vollmer [52], $3.5 \mathrm{meV}$ from White et al. [56], $3.7 \mathrm{meV}$ from Ellis et al. [57], and $3.65 \mathrm{meV}$ from Hutson and Schwartz [24].

[56] J. D. White, J. V. Lakin, M. A. Strauss, and R. D. Diehl, J. Chem. Phys. 101, 4445 (1994).

[57] T. H. Ellis, G. Scoles, U. Valbusa, H. Jónsson, and J. H. Weare, Surf. Sci. 155, 499 (1985)

[58] The effective number of $\mathbf{G}$ channels in their diffraction calculation at $E_{i}=63.77 \mathrm{meV}$ is 301 and $N_{\mathbf{G}}=349$ in ours.

[59] A. D. Novaco and J. P. McTague, Phys. Rev. Lett. 38, 1286 (1977); J. P. McTague and A. D. Novaco, Phys. Rev. B 19, 5299 (1979).
[60] A. Šiber, B. Gumhalter, A. P. Graham, and J. P. Toennies, Phys. Rev. B 63, 115411 (2001).

[61] C. Ramseyer, C. Girardet, P. Zeppenfeld, J. Goerge, M. Büchel, and G. Comsa, Surf. Sci. 313, 251 (1994).

[62] P. Zeppenfeld, M. Büchel, J. Goerge, R. David, G. Comsa, C. Ramseyer, and C. Girardet, Surf. Sci. 366, 1 (1996), Sec. 2.2.

[63] Unpublished work of Bertino, Graham, and Toennies, cited at Ref. 77 of Ref. [7]. The misalignment is noted in the Introduction to R. Gerlach, A. P. Graham, J. P. Toennies, and H. Weiss, J. Chem. Phys. 109, 5319 (1998).

[64] This case has the striking result that $Q I_{o}^{-} / Q I_{o}^{+}=1.25$, i.e., the energy gain strength for the phonon of energy $\omega=0.719 \mathrm{meV}$ is larger than that of the energy loss.

[65] P. Cantini and R. Tatarek, Surf. Sci. 114, 471 (1982).

[66] U. Garibaldi, A. C. Levi, R. Spadacini, and G. E. Tommei, Surf. Sci. 48, 649 (1975).

[67] P. Kraus, M. Mayrhofer-Reinhartshuber, C. Gösweiner, F. Apolloner, S. Miret-Artés, and W. E. Ernst, Surf. Sci. 630, 208 (2014).

[68] H. Kasai and W. Brenig, Z. Phys. B 59, 429 (1985); A. C. Levi, J. Phys.: Condens. Matter 21, 405004 (2009).

[69] R. D. Diehl, T. Seyller, M. Caragiu, G. S. Leatherman, N. Ferralis, K. Pussi, P. Kaukasoina, and M. Lindroos, J. Phys.: Condens. Matter 16, S2839 (2004).

[70] The Debye-Waller exponent $W_{\text {DW }}$ depends on $\mathbf{q}$ because the angle of incidence $\theta_{i}$ depends on the mode $\mathbf{q}$ that is excited in the scan-curve geometry of the scattering experiments. This parametric dependence is not included in the notation, but it is included in evaluating $W_{\mathrm{DW}}(T)$.

[71] See, e.g., J. S. Becker, R. D. Brown, E. Johansson, N. S. Lewis, and S. J. Sibener, J. Chem. Phys. 133, 104705 (2010); Eqs. (5)-(7). 